DRAFT VERSION MARCH 4, 2021

Typeset using LATEX twocolumn style in AASTeX63

\title{
The Impact of Stellar Clustering on the Observed Multiplicity and Orbital Periods of Planetary Systems
}

\author{
Steven N. Longmore, ${ }^{1}$ Mélanie Chevance, ${ }^{2}$ And J. M. Diederik Kruijssen ${ }^{2}$ \\ ${ }^{1}$ Astrophysics Research Institute, Liverpool John Moores University, IC2, Liverpool Science Park, 146 Brownlow Hill, Liverpool L3 5RF, UK \\ ${ }^{2}$ Astronomisches Rechen-Institut, Zentrum für Astronomie der Universität Heidelberg, Mönchhofstraße 12-14, D-69120 Heidelberg, Germany
}

(Received 2020 October 22; Revised 2021 March 1; Accepted 2021 March 2)

Submitted to ApJ Letters

\begin{abstract}
It has recently been shown that stellar clustering plays an important role in shaping the properties of planetary systems. We investigate how the multiplicity distributions and orbital periods of planetary systems depend on the 6D phase space density of stars surrounding planet host systems. We find that stars in high stellar phase space density environments (overdensities) have a factor 1.6-2.0 excess in the number of single planet systems compared to stars in low stellar phase space density environments (the field). The multiplicity distribution of planets around field stars is much flatter (i.e. there is a greater fraction of multi-planet systems) than in overdensities. This result is primarily driven by the combined facts that: (i) 'hot Jupiters' (HJs) are almost exclusively found in overdensities; (ii) HJs are predominantly observed to be single-planet systems. Nevertheless, we find that the difference in multiplicity is even more pronounced when only considering planets in the Kepler sample, which contains few HJs. This suggests that the Kepler dichotomy - an apparent excess of systems with a single transiting planet - plausibly arises from environmental perturbations. In overdensities, the orbital periods of single-planet systems are smaller than orbital periods of multiple-planet systems. As this difference is more pronounced in overdensities, the mechanism responsible for this effect may be enhanced by stellar clustering. Taken together, the pronounced dependence of planetary multiplicity and orbital period distributions on stellar clustering provides a potentially powerful tool to diagnose the impact of environment on the formation and evolution of planetary systems.
\end{abstract}

Keywords: solar-planetary interactions - exoplanet systems - exoplanet formation — planet formation star formation — stellar dynamics

\section{INTRODUCTION}

The comparison of planetary properties between singleand multiple-planet systems has long been used to probe the formation and evolution of planetary systems (Wright et al. 2009; Lissauer et al. 2011; Winn \& Fabrycky 2015; Mulders et al. 2018; Weiss et al. 2018a,b). Motivated by the rich variety of exoplanetary systems observed with the Kepler mission (Borucki et al. 2010, 2011), the similarity of the host-star properties, planet radii, and radius valley for single-planet and multi-planet systems has been used to infer they have a common origin (Weiss et al. 2018a; Rogers $\&$ Owen 2020). However, since the early days of Kepler, it has been known that using a single population of planetary systems that matches the higher multiplicities simultaneously underpredicts the number of singly transiting systems (Lis-

Corresponding author: Steven N. Longmore

s.n.longmore@1jmu.ac.uk sauer et al. 2011; Hansen \& Murray 2013; Ballard \& Johnson 2016). The apparent excess of systems with a single transiting planet - known as the Kepler dichotomy (e.g. Johansen et al. 2012) - places important constraints on the degree to which all planets may share a common origin.

Different scenarios have been proposed to explain the origin of the Kepler dichotomy (see e.g. He et al. 2019, 2020, and references therein). Some scenarios attempt to assess whether all observed planet properties can be described by a single underlying population. Such studies invoke an intrinsically high fraction of single systems (Fang \& Margot 2012; Sandford et al. 2019) or a strong anti-correlation between the mutual inclination scale and the multiplicity of each system (Zhu 2020). Other scenarios assert that more than one planet population is required, between which the orbital properties may vary (e.g. the mutual inclination, see Mulders et al. 2018; He et al. 2019). Observations show an anti-correlation between planet multiplicity and their dynamical excitation (e.g. Morton \& Winn 2014a; Van Eylen 
et al. 2019), which points towards a scenario in which highmultiplicity systems can became dynamically unstable and lose some of their planets (see e.g. Zinzi \& Turrini 2017), leaving behind dynamically excited, compact systems. In this case, most observed 'single' planets are, in fact, part of misaligned multiple-planet systems (He et al. 2020). These scenarios suggest perturbations of planetary systems may play a role in shaping the Kepler dichotomy.

Following the idea that the Kepler dichotomy may result from perturbations, we assess the role that the ambient stellar clustering plays in shaping the multiplicity and orbital properties of planetary systems (also see e.g. Cai et al. 2018). We take the sample of known exoplanets and use the ambient stellar phase space density obtained with Gaia (Gaia Collaboration et al. 2016, 2018) to divide the sample into low and high ambient stellar phase space densities (Winter et al. 2020), which we refer to as planets residing in the 'field' and in 'overdensities', respectively. These subsamples are considered as reflecting environments of low and high degrees of perturbation.

This investigation mirrors those in a set of companion papers, where we investigate the impact of stellar clustering on the orbital period distribution of planets and the incidence of hot Jupiters (Winter et al. 2020) and on the correlation between the properties of adjacent planets (Chevance et al. 2021, i.e. 'peas in a pod', Weiss et al. 2018a), as well as its role in turning sub-Neptunes into super-Earths (Kruijssen et al. 2020, i.e. driving them across the 'radius valley', Fulton et al. 2017). These findings demonstrate that stellar clustering has a major impact on the architectures of planetary systems, plausibly through external photoevaporation or dynamical perturbations (e.g. Winter et al. 2020).

In this Letter, we investigate the extent to which the Kepler dichotomy and the orbital properties of planets in single and multiple systems may arise from such environmental perturbations.

We find an excess of single-planet systems in overdensities, strongly suggesting that the Kepler dichotomy might indeed arise due to the impact of the large-scale stellar environment. The single-planet population in overdensities is characterised by shorter orbital periods than the multi-planet population, suggesting that the perturbations cause the remaining planet to migrate to tighter orbits.

\section{METHOD}

The subsequent analysis relies on the division of known exoplanetary systems into field and overdensity systems by Winter et al. (2020). The primary exoplanet sample is drawn from the 'catch-all' NASA Exoplanet Archive (2020), which contains a heterogeneous mix of exoplanet detections from ground-based and space-based facilities using a range of different observational techniques. To avoid issues with various observational biases related to sample selection, the Winter et al. (2020) method explicitly does not search for correlations, or look for relationships between planet properties, within the database alone. Instead, the method splits exoplanet host stars in the database in a carefully controlled way using an independent dataset (Gaia), such that the inherent biases, selection effects, etc., in the archive are the same (within the inherent uncertainties) between the split populations. Through subsequent Monte Carlo experiments, we have verified that any residual biases when making the split are not the cause of the difference in planetary properties between the sub-samples (see, e.g., Figure 3 and Extended Data Figures 3, 4, 5, 7, 8 in Winter et al. 2020).

The Winter et al. (2020) sample contains 1522 planets orbiting 1137 stars, drawn from the NASA Exoplanet Archive (2020). Winter et al. (2020) calculated the relative, six-dimensional position-velocity phase space density of every exoplanet host star from Gaia's second data release (Gaia Collaboration et al. 2018) for which radial velocities are also available (1522 out of 4141 exoplanets), as well as for up to 600 neighbouring stars $\left(N_{\mathrm{ss}}\right)$ within $40 \mathrm{pc}$ of each exoplanet host. For most exoplanet hosts, the resulting phase space density distributions of nearby stars is well described with a double lognormal. To quantify this, Winter et al. (2020) determine the probability $P_{\text {null }}$ that the phase space density distribution of each stellar host neighbourhood is drawn from a single lognormal distribution.

As described in Winter et al. (2020), we first remove stars from this sample for which it is not possible to reliably decompose their phase space distributions into low- and highdensity components. This can be either due to a low number of neighbours or because their local phase space density distribution is not bimodal (i.e. we remove stars for which $\left.P_{\text {null }} \geq 0.05 \& N_{\mathrm{ss}}<400\right)$. As exoplanet architectures correlate with the mass and age of the host star (Kennedy \& Wyatt 2013; Winn \& Fabrycky 2015), we further only include stars with ages $1-4.5 \mathrm{Gyr}$ and masses $0.7-2 \mathrm{M}_{\odot}$, to ensure that the low- and high-density sub-samples have similar distributions in these properties. These cuts leave 399 stellar systems in our fiducial reliable sample.

For these remaining host stars, Winter et al. (2020) perform a double lognormal decomposition of the local phase space density distribution, identifying a low- and a highdensity component. The decomposition yields the probability $P_{\text {high }}$ (respectively $P_{\text {low }}=1-P_{\text {high }}$ ) that an individual host star lies in a phase space overdensity (respectively underdensity). We subsequently split the sample into a high $\left(P_{\text {high }}>0.84\right)$ and a low $\left(P_{\text {low }}>0.84\right)$ phase space density sample. The choice of a $1 \sigma$ threshold of 0.84 in $P_{\text {high }}$ and $P_{\text {low }}$ represents a compromise between obtaining a large sample and minimising misclassification. The general con- 
clusions of the paper are robust against sensible changes in this threshold.

Table 1 shows the number of stars and the planet multiplicity distributions for the full reliable sample and different low- and high-density sub-samples, which we refer to as the 'field' and 'overdensities', respectively. Table 2 shows the mean distribution of host stellar mass, host stellar metallicity, host stellar age, distance from the Sun and the number of systems in which at least one planet has been detected through transit and radial velocity (RV) measurements, for the sub-set of the samples in Table 1 with statistically significant differences in the single-to-multiple planet ratios between the field and overdensities.

\section{RESULTS}

We break up the analysis into three parts. Firstly, in $\S 3.1$ and $\S 3.2$ we focus on planetary multiplicity, as well as on their orbital periods and resonances, respectively, using the full Winter et al. (2020) sample (i.e. the 'Field' and 'Overdensities' samples in the top rows of Tables 1 and 2). The Winter et al. (2020) catalogue represents the largest possible sample of field and overdensities, at the expense of the highest degree of sample heterogeneity in terms of e.g. selection and detection method. In $\S 3.3$, we focus on sub-samples of the Winter et al. (2020) catalogue that are drawn from individual exoplanet surveys to reduce the data heterogeneity, at the expense of sample size.

\subsection{Multiplicity: full Winter et al. (2020) sample}

We start by investigating the multiplicity of the 'Field' and 'Overdensities' samples from the full Winter et al. (2020) catalogue. Winter et al. (2020) ruled out stellar mass, metallicity and distance as systematic sources of bias between the field and overdensity samples. It is therefore unlikely that observational biases in these parameters would manifest themselves as differences in planet multiplicity (or orbital period (ratios), §3.2) between the field and overdensity samples.

Table 1 shows there are clear differences in the distribution of planet multiplicity between the field and overdensities. In particular, overdensities have a greater fraction of systems with only one detected planet. The final column of Table 1 shows overdensities have a factor 1.6 excess of single planet systems compared to the field. Figure 1 visualises the planet multiplicity distributions of both sub-samples. The planet multiplicity distribution of stars in the field sample is significantly flatter, i.e. a greater fraction of systems in the field have more than one planet.

Table 2 also shows that there are differences in the fraction of planets in the field and overdensities that have been detected through transit and RV observations. The most notable difference is that only $35 \%$ of the field star systems have planets detected through transits compared to $63 \%$ for over- densities. Given that transit and RV observations are sensitive to detecting planets in different mass and orbital period regimes, it is possible that this imbalance in detection method may imprint a bias in the planetary multiplicity, orbital period and orbital period ratio between the field and overdensity samples.

To estimate the potential bias due to imbalances in observational detection methods, we split the overdensity ${ }^{1}$ sample into systems where all planets are exclusively detected through transits (transit-only sample) and those where all planets are exclusively detected through radial velocity measurements (RV-only sample). These sub-samples contain 67 and 89 systems, respectively. We repeat the entire analysis in $\S 3.1$ and $\S 3.2$ using the transit-only and RV-only samples to determine how robust the identified differences between the full Winter et al. (2020) field and overdensity samples are against detection method bias. We then focus our discussion on results that are found to be robust, defined such that any bias would act in the opposite direction of the identified trends, and that correcting for this bias would either strengthen the results or leave them unchanged, rather than weakening them. We explicitly refer to the results of the detection method bias test after each analysis step below.

Repeating the multiplicity analysis on the RV-only and transit-only samples shows that the RV-only samples have a steeper drop in multiplicity, i.e. they are more likely to detect single-planet systems. As the field sample planet population is dominated by RV measurements and recovers a smaller fraction of single-planet systems than overdensities, we infer that the difference in multiplicity distribution seen between the field and overdensity systems cannot be explained by a detection method bias, and that accounting for this bias would only strengthen the above results.

To illustrate this point, we formulate an approximate correction of the field and overdensity samples for the detection method bias. We measure the number of systems per multiplicity value in the RV-only and transit-only samples, and normalise them by the total number of systems in the RV-only sample $\left(N_{\mathrm{ov}, \mathrm{rv}}=89\right)$ and in the transit-only sample $\left(N_{\mathrm{ov}, \mathrm{tr}}=67\right)$. For a given multiplicity value, $i$, the normalised fraction of systems is then $f_{i, \mathrm{rv}}$ and $f_{i, \mathrm{tr}}$ for the RV-only and transit-only samples, respectively. Relative to a sample containing an equal number of transit and radial velocity detections, the bias of each individual detection method in terms of the fraction of systems per multiplicity value is defined as,

$$
\delta_{i, \mathrm{rv}}=\frac{1}{2}\left(f_{i, \mathrm{rv}}-f_{i, \mathrm{tr}}\right) \quad \text { and } \quad \delta_{i, \mathrm{tr}}=\frac{1}{2}\left(f_{i, \mathrm{tr}}-f_{i, \mathrm{rv}}\right) .
$$

\footnotetext{
${ }^{1}$ A similar experiment cannot be performed for the field sample due to the low number of field systems.
} 

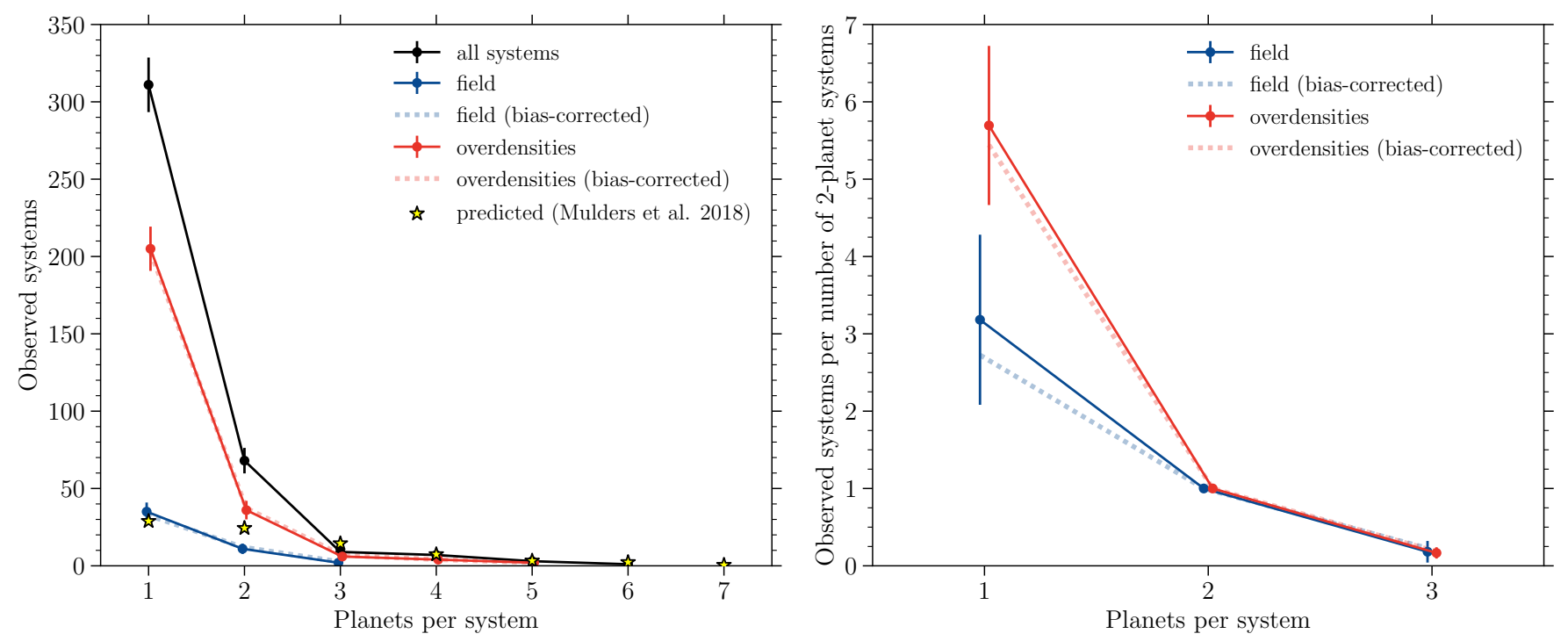

Figure 1. Planetary system multiplicity distributions from the full Winter et al. (2020) sample. The left panel shows multiplicity distributions for the full reliable sample (black, 'all systems'), as well as the low-density (blue, 'field'), and high-density (red, 'overdensities') sub-samples. The shaded blue and red dotted lines show the values corrected for the method detection bias (see the text). Error bars represent the Poissonian $(\sqrt{N})$ uncertainties on the data points. Stars show the co-planar multiplicity distribution predicted by Mulders et al. (2018, their fig. 11), scaled down by a factor of 30 to enable an easier comparison to the samples in this work. The right panel shows the same planet multiplicity distributions, but this time each sample has been normalised to the number of systems with two planets. Both panels show that the planet multiplicity distribution of systems in the 'field' and 'overdensities' differ considerably. While overdensities are greatly dominated by single-planet systems, the planet multiplicity distribution of stars in the field sample is significantly flatter and more closely matches the model predictions.
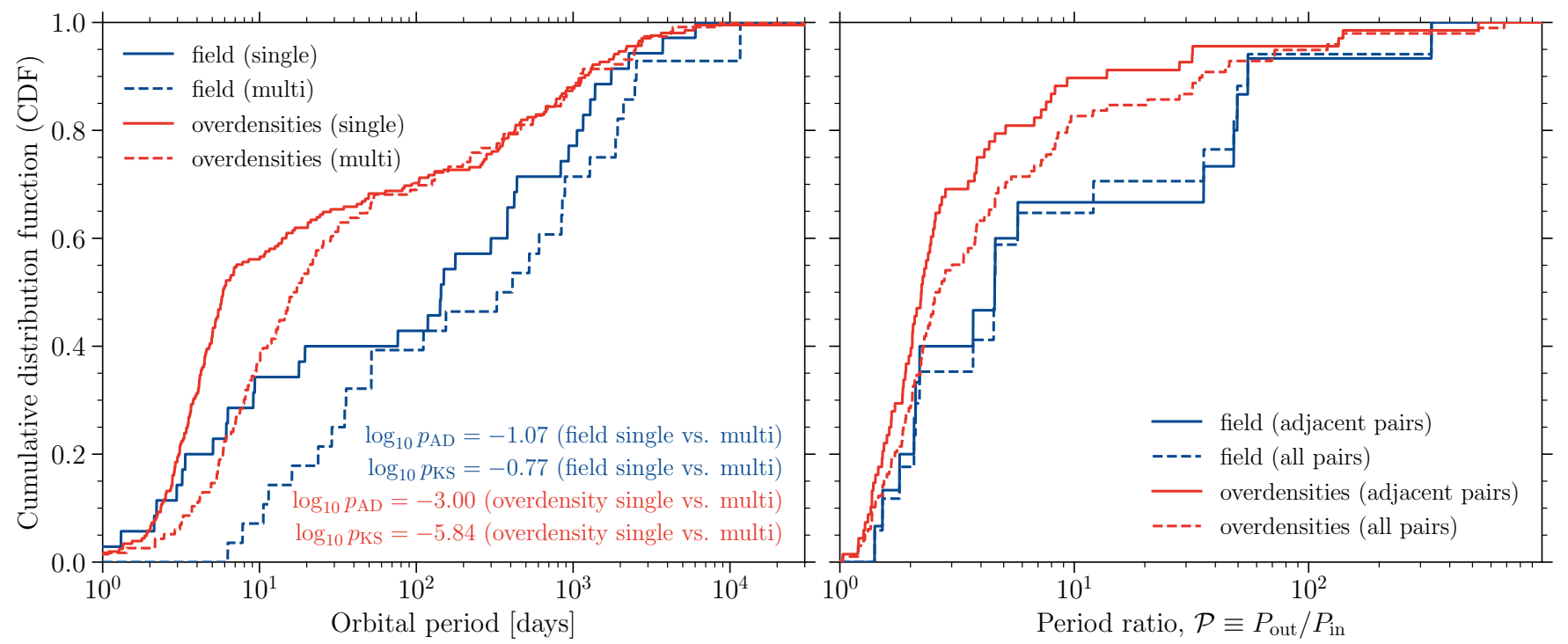

Figure 2. Orbital period demographics in field and overdensity systems for the full Winter et al. (2020) sample. The left panel shows the cumulative distribution functions of orbital periods for planets around stars in overdensities (red) and the field (blue). The solid lines show the distribution for stellar systems with only a single planet ('single'), while the dashed lines show the distribution for stellar systems with more than one planet ('multi'). The $p_{\mathrm{KS}}$ and $p_{\mathrm{AD}}$ values in the bottom right corner show the results of two-sample Kolmogorov-Smirnov and Anderson-Darling tests, respectively, assessing the null hypothesis that the two samples listed in parentheses after the $p_{\mathrm{KS}}$ and $p_{\mathrm{AD}}$ values are drawn from the same population. This is ruled out at high confidence for the overdensity sample, for which the orbital periods of single-planet systems are significantly smaller than those of multiple-planet systems. For the field sample, the period distributions of single and multi-planet systems are statistically indistinguishable. The right panel shows the cumulative distribution function of pairs of planets within the same system with period ratio $\mathcal{P}=P_{\text {out }} / P_{\text {in }}$, where $P_{\text {out }}$ and $P_{\text {in }}$ are the periods of the outer and inner planet, respectively. Dashed lines show all planet pairs and solid lines show only adjacent planet pairs. Blue and red show the field and overdensity samples, respectively. Planetary systems in overdensities may be somewhat more closely packed than in the field, but not at high significance. 


\begin{tabular}{|c|c|c|c|c|c|c|c|c|}
\hline (1) & (2) & (3) & (4) & (5) & (6) & (7) & (8) & (9) \\
\hline Sample & $\begin{array}{l}\text { Total number } \\
\text { of systems }\end{array}$ & $N\left(n_{p}=1\right)$ & $\begin{array}{c}\text { Number of syst } \\
N\left(n_{p}=2\right)\end{array}$ & $\begin{array}{c}\text { ems }(N) \text { with d } \\
N\left(n_{p}=3\right)\end{array}$ & $\begin{array}{l}\text { ifferent numbers } \\
N\left(n_{p}=4\right)\end{array}$ & $\begin{array}{l}\text { of planets }\left(n_{p}\right) \\
N\left(n_{p}=5\right)\end{array}$ & $N\left(n_{p}=6\right)$ & $\begin{array}{c}\text { Ratio: sing. to mult. } \\
\qquad \begin{array}{c}N\left(n_{p}=1\right) / \\
N\left(n_{p}>1\right)\end{array}\end{array}$ \\
\hline $\begin{array}{l}\text { Full reliable sample } \\
\text { ('All') }\end{array}$ & 399 & 311 & 68 & 9 & 7 & 3 & 1 & $3.53 \pm 0.43$ \\
\hline $\begin{array}{l}\text { Low density } \\
\text { ('Field') }\end{array}$ & 48 & 35 & 11 & 2 & 0 & 0 & 0 & $2.69 \pm 0.87$ \\
\hline $\begin{array}{l}\text { High density } \\
\text { ('Overdensities') }\end{array}$ & 253 & 205 & 36 & 6 & 4 & 2 & 0 & $4.27 \pm 0.68$ \\
\hline $\begin{array}{l}\text { Low density, no hot Jupiters } \\
\text { ('Field, no HJ') }\end{array}$ & 40 & 27 & 11 & 2 & 0 & 0 & 0 & $2.08 \pm 0.70$ \\
\hline $\begin{array}{l}\text { High density, no hot Jupiters } \\
\text { ('Overdensities, no HJ') }\end{array}$ & 157 & 112 & 33 & 6 & 4 & 2 & 0 & $2.48 \pm 0.44$ \\
\hline $\begin{array}{c}\text { Low density, Kepler \& K2 } \\
\text { ('Field, Kepler/K2') }\end{array}$ & 8 & 5 & 2 & 1 & 0 & 0 & 0 & $1.67 \pm 1.22$ \\
\hline $\begin{array}{l}\text { High density, Kepler \& K2 } \\
\text { ('Overdens, Kepler/K2') }\end{array}$ & 79 & 52 & 19 & 5 & 3 & 0 & 0 & $1.93 \pm 0.46$ \\
\hline $\begin{array}{l}\text { Low density, Kepler-only } \\
\text { ('Field, Kepler') }\end{array}$ & 8 & 5 & 2 & 1 & 0 & 0 & 0 & $1.67 \pm 1.22$ \\
\hline $\begin{array}{l}\text { High density, Kepler-only } \\
\text { ('Overdens, Kepler') }\end{array}$ & 72 & 46 & 18 & 5 & 3 & 0 & 0 & $1.77 \pm 0.43$ \\
\hline $\begin{array}{l}\text { Low density, WASP+HAT } \\
\text { ('Field, WASP+HAT') }\end{array}$ & 4 & 4 & 0 & 0 & 0 & 0 & 0 & - \\
\hline $\begin{array}{l}\text { High density, WASP+HAT } \\
\text { ('Overdens, WASP+HAT') }\end{array}$ & 58 & 56 & 2 & 0 & 0 & 0 & 0 & $28.00 \pm 20.15$ \\
\hline $\begin{array}{l}\text { Low density, Kepler (CKS) } \\
\text { ('Field, Kepler/CKS') }\end{array}$ & 5 & 2 & 2 & 0 & 1 & 0 & 0 & $0.67 \pm 0.61$ \\
\hline $\begin{array}{l}\text { High density, Kepler (CKS) } \\
\text { ('Overdens, Kepler/CKS') }\end{array}$ & 60 & 38 & 13 & 5 & 3 & 0 & 1 & $1.73 \pm 0.46$ \\
\hline $\begin{array}{c}\text { Low density, Kepler (CKS), } \\
\mathrm{M}_{*}>0.5 \mathrm{M}_{\odot} \\
\text { ('Field, Kep/CKS }, 0.5 \mathrm{M}_{\odot} \text { ') }\end{array}$ & 6 & 2 & 3 & 0 & 1 & 0 & 0 & $0.50 \pm 0.43$ \\
\hline $\begin{array}{c}\text { High density, Kepler (CKS), } \\
\mathrm{M}_{*}>0.5 \mathrm{M}_{\odot} \\
\text { ('Overdens, Kep/CKS, } 0.5 \mathrm{M}_{\odot} \text { ') }\end{array}$ & 69 & 44 & 15 & 5 & 4 & 0 & 1 & $1.76 \pm 0.44$ \\
\hline $\begin{array}{c}\text { Low density, Kepler (CKS), } \\
0.5 \mathrm{M}_{\odot}<\mathrm{M}_{*}<1.0 \mathrm{M}_{\odot} \\
\text { ('Field, Kep/CKS, } 0.5-1.0 \mathrm{M}_{\odot}{ }^{\prime} \text { ) }\end{array}$ & 6 & 2 & 3 & 0 & 1 & 0 & 0 & $0.50 \pm 0.43$ \\
\hline $\begin{array}{c}\text { High density, Kepler }(\mathrm{CKS}) \\
0.5 \mathrm{M}_{\odot}<\mathrm{M}_{*}<1.0 \mathrm{M}_{\odot} \\
\text { ('Overdens, Kep/CKS, } 0.5-1.0 \mathrm{M}_{\odot} \text { ') }\end{array}$ & 27 & 18 & 7 & 0 & 1 & 0 & 1 & $2.00 \pm 0.82$ \\
\hline
\end{tabular}

Table 1. The number of stellar systems and the planet multiplicity distributions for different data samples. The second column gives the total number of planetary systems in each of the samples. Columns 3 to 8 give the number of systems in each of the samples with the specified number of detected planets. The final column gives the ratio (and associated Poisson uncertainty) of the number of single and multiple planetary systems. All samples above the double horizontal line use the stellar parameters from the NASA Exoplanet Archive (NASA Exoplanet Archive 2020). The Kepler-only samples below the double horizontal line use stellar parameters from the California-Kepler Survey (CKS, taken from Fulton \& Petigura 2018). Rows with $\mathrm{M}_{*}>0.5 \mathrm{M}_{\odot}$ use a lower mass cutoff of $0.5 \mathrm{M}_{\odot}$ for the host stellar mass, rather than $0.7 \mathrm{M}_{\odot}$ as for all other samples. The final row uses an upper mass cutoff of $1.0 \mathrm{M}_{\odot}$, rather than $2.0 \mathrm{M}_{\odot}$ for all other samples. The WASP + HAT rows are included to show that ground-based data currently do not have large enough samples of planets in low-density environments to enable a meaningful comparison of multiplicities. 


\begin{tabular}{|c|c|c|c|c|c|c|c|}
\hline $\begin{array}{c}(1) \\
\text { Sample }\end{array}$ & $\begin{array}{c}(2) \\
\text { Stellar } \\
\text { mass } \\
{\left[\mathrm{M}_{\odot}\right]} \\
\end{array}$ & $\begin{array}{c}(3) \\
\text { Stellar } \\
\text { metallicity } \\
\text { [dex] } \\
\end{array}$ & $\begin{array}{c}(4) \\
\text { Stellar } \\
\text { age } \\
{[\mathrm{Gyr}]} \\
\end{array}$ & $\begin{array}{c}(5) \\
\text { Distance } \\
\text { from sun } \\
{[\mathrm{pc}]} \\
\end{array}$ & $\begin{array}{c}\text { (6) } \\
\text { Number } \\
\text { of } \\
\text { systems } \\
\end{array}$ & $\begin{array}{c}\text { (7) } \\
\text { Num. systems } \\
\text { with } \geq 1 \text { transit } \\
\text { detections } \\
\end{array}$ & $\begin{array}{c}\text { (8) } \\
\text { Num. systems } \\
\text { with } \geq 1 \text { radial } \\
\text { velocity detections }\end{array}$ \\
\hline $\begin{array}{l}\text { Low density } \\
\text { ('Field') }\end{array}$ & $1.09_{-0.24}^{+0.41}$ & $0.08_{-0.28}^{+0.18}$ & $3.2_{-1.3}^{+0.6}$ & $65.0_{-33.0}^{+197.0}$ & 48 & 17 & 40 \\
\hline $\begin{array}{c}\text { High density } \\
\text { ('Overdensities') }\end{array}$ & $1.2_{-0.25}^{+0.21}$ & $0.09_{-0.13}^{+0.15}$ & $2.7_{-1.0}^{+1.3}$ & $218.0_{-167.0}^{+214.0}$ & 253 & 160 & 182 \\
\hline $\begin{array}{c}\text { Low density, Kepler (CKS) } \\
\text { ('Field, Kepler/CKS') }\end{array}$ & $0.78_{-0.05}^{+0.35}$ & $0.02_{-0.33}^{+0.15}$ & $3.8_{-0.6}^{+0.5}$ & $172.0_{-19.0}^{+60.0}$ & 5 & 5 & 1 \\
\hline $\begin{array}{l}\text { High density, Kepler (CKS) } \\
\text { ('Overdens, Kepler/CKS') }\end{array}$ & $1.08_{-0.14}^{+0.18}$ & $0.07_{-0.1}^{+0.13}$ & $2.9_{-1.0}^{+1.1}$ & $389.0_{-144.0}^{+137.0}$ & 60 & 60 & 14 \\
\hline $\begin{array}{l}\text { Low density, Kepler (CKS), } \\
\qquad \mathrm{M}_{*}>0.5 \mathrm{M}_{\odot} \\
\text { ('Field, Kepler/CKS, } 0.5 \mathrm{M}_{\odot} \text { ') }\end{array}$ & $0.75_{-0.06}^{+0.33}$ & $-0.12_{-0.19}^{+0.22}$ & $3.8_{-0.6}^{+0.5}$ & $172.0_{-19.0}^{+60.0}$ & 6 & 6 & 1 \\
\hline $\begin{array}{c}\text { High density, Kepler (CKS), } \\
\qquad \mathrm{M}_{*}>0.5 \mathrm{M}_{\odot} \\
\text { ('Overdens, Kepler/CKS }, 0.5 \mathrm{M}_{\odot}{ }^{\prime} \text { ) }\end{array}$ & $1.07_{-0.13}^{+0.18}$ & $0.07_{-0.1}^{+0.12}$ & $2.7_{-1.4}^{+1.3}$ & $354.0_{-121.0}^{+172.0}$ & 69 & 69 & 15 \\
\hline $\begin{array}{c}\text { High density, Kepler (CKS), } \\
0.5 \mathrm{M}_{\odot}<\mathrm{M}_{*}<1.0 \mathrm{M}_{\odot} \\
\text { ('Overdens, Kepler/CKS, } 0.5-1.0 \mathrm{M}_{\odot}{ }^{\prime} \text { ) }\end{array}$ & $0.94_{-0.12}^{+0.04}$ & $0.02_{-0.06}^{+0.07}$ & $1.7_{-0.7}^{+1.5}$ & $285.0_{-96.0}^{+73.0}$ & 27 & 27 & 6 \\
\hline
\end{tabular}

Table 2. Investigation of potential observational biases in the properties of different field and overdensity samples (see Table 1 for sample details). Columns 2 to 5 show the mean $\pm 1 \sigma$ distribution of host stellar mass, host stellar metallicity, host stellar age and distance from the Sun. Columns 6 to 8 show the total number of stellar systems and the number of systems in which at least one planet has been detected through transit and radial velocity measurements, respectively.

We then weigh these biases by the numbers of systems that are detected only by radial velocities $\left(N_{\mathrm{rv}}\right)$ or transit $\left(N_{\mathrm{tr}}\right)$ in each of the field and overdensity samples to correct the measurements as

$$
f_{i} \rightarrow f_{i}-\left(\frac{N_{\mathrm{rv}}}{N_{\mathrm{rv}}+N_{\mathrm{tr}}} \delta_{i, \mathrm{rv}}+\frac{N_{\mathrm{tr}}}{N_{\mathrm{rv}}+N_{\mathrm{tr}}} \delta_{i, \mathrm{tr}}\right) .
$$

The number of systems per multiplicity value $i$ is then this corrected fraction $f_{i}$ multiplied by the total number of systems in the sample (field or overdensity). The results are shown as shaded dotted lines in Figure 1 and clearly show that correcting for detection method biases would strengthen our finding that overdensities show an excess of single-planet systems compared to the field, increasing the relative excess of single-planet systems in overdensities relative to the field from 1.6 to 2.0. This suggests that future work repeating this analysis on larger and more homogeneous samples has the potential to find an even stronger multiplicity distribution difference between systems in the field and in overdensities.

\subsection{Orbital period and resonances: full sample}

We now turn to a discussion of the orbital period distributions as a function of planetary multiplicity for the full Winter et al. (2020) sample. The left-hand panel of Figure 2 shows the cumulative distribution functions of the orbital period for the field and overdensities, split into systems with only one planet ('single') and more than one planet ('multi'). We conduct two-sample Anderson-Darling (AD) and KolmogorovSmirnov (KS) tests assessing whether the orbital period distributions of both single- and multi-planet systems in the field and overdensities are drawn from the same population. As reported in Winter et al. (2020), the orbital periods of planets in the field are significantly larger than in overdensities. The new analysis here shows that this holds for both single and multiple planet systems. However, repeating this analysis with RV-only and transit-only samples shows that there is a strong bias in the RV-only sample towards detecting planets with longer orbital periods. As planets detected around field stars are dominated by RV observations, the observed increase in orbital period for planets in the field compared to overdensities may be affected by detection method bias. 
We therefore refrain from a quantitative comparison of the orbital period distributions between the field and overdensity samples.

Instead, we focus on comparing the orbital period distributions between the single- and multiple-planet samples within the field and overdensities independently. This ensures the analysis is not affected by detection method bias. The resulting $p_{\mathrm{AD}}$ and $p_{\mathrm{KS}}$-values, shown in Figure 2, strongly rule out the null hypothesis that the orbital periods of single and multiple planet systems in overdensities are drawn from the same parent distribution. In overdensities, the observed orbital periods of single-planet systems are significantly smaller than orbital periods of multiple-planet systems. While the orbital period distributions of single-planet systems in the field also appear smaller than for multipleplanet systems, the $p_{\mathrm{AD}}$ and $p_{\mathrm{KS}}$-values cannot rule out that they are drawn from the same population.

For all systems with more than one planet, we then derive the ratio of orbital periods, $\mathcal{P}$, of the outer $\left(P_{\text {out }}\right)$ to inner $\left(P_{\text {in }}\right)$ planet, $\mathcal{P} \equiv P_{\text {out }} / P_{\text {in }}$, for all planet pairs within that system. Figure 2 shows the cumulative distribution function of $\mathcal{P}$ for the field and overdensity samples, for all planet pairs and adjacent planet pairs.

The period ratio for planet pairs in overdensities appears systematically smaller than for the field, with a slightly more pronounced offset for neighbouring planets than for all planet pairs. We conduct a two-sample KS test against the null hypothesis that the field and overdensity samples are drawn from the same $\mathcal{P}$ distribution. Despite the apparent offset between the field and overdensity period ratios, the resulting $p_{\mathrm{KS}}\left(p_{\mathrm{AD}}\right)$ values of $0.14(0.06)$ for adjacent pairs and $0.31(0.25)$ for all pairs mean the offset is not statistically significant enough to rule out the null hypothesis. In addition, repeating the analysis with the RV-only and transit-only samples suggests a small potential bias of RV-only samples towards longer period ratios. Lissauer et al. (2011) report a similar offset in their comparison of Kepler and RV samples. As the field planet population is dominated by RV measurements, the apparent increase in $\mathcal{P}$ compared to overdensities may therefore be affected by detection method bias.

Finally, we investigate whether there are any differences in the distribution of orbital period ratios of planetary system pairs in relation to mean motion resonances (MMRs; orbital period ratios that are nearly equal to ratios of small integers) between the field and overdensity samples. Following Lissauer et al. (2011), we use the variable $\zeta$ to measure the difference between an observed period ratio and nearby MMRs. In order to treat all neighbourhoods equally, $\zeta$ ranges between -1 and 1 in each neighbourhood.
For first-order MMRs (for which the orbital period ratios are $j: j-1$, i.e. $2: 1,3: 2,4: 3, \ldots), \zeta_{1}$ is given by

$$
\zeta_{1}=3\left[\frac{1}{\mathcal{P}-1}-\text { Round }\left(\frac{1}{\mathcal{P}-1}\right)\right],
$$

where 'Round' returns the nearest integer. For second order MMRs (for which the orbital period ratios are $j: j-2$, i.e., $3: 1,4: 2,5: 3, \ldots), \zeta_{2}$ is given by the analogous expression

$$
\zeta_{2}=3\left[\frac{2}{\mathcal{P}-1}-\text { Round }\left(\frac{2}{\mathcal{P}-1}\right)\right] .
$$

We conduct two-sample KS and AD tests against the null hypothesis that the field and overdensity samples are drawn from the same $\zeta_{1}$ and $\zeta_{2}$ distributions. When including all planet pairs, there are no statistically significant differences in either the $\zeta_{1}$ or $\zeta_{2}$ distributions between the field and overdensity samples. However, when only considering adjacent planet pairs, we do find a statistically significant difference.

Figure 3 shows cumulative distribution functions of the relative proximity of the orbital period ratios of adjacent planet pairs to MMRs, for the field and overdensity subsamples. The $p_{\mathrm{KS}}\left(p_{\mathrm{AD}}\right)$ value of $0.32(0.20)$ shows that there is no statistically significant difference between the $\zeta_{1}$ distributions of the field and overdensity samples. Given our null hypothesis threshold, $p_{\text {ref }}=0.05$, the $p_{\mathrm{KS}}\left(p_{\mathrm{AD}}\right)$ value of $8.9 \times 10^{-3}(0.04)$ suggests that the $\zeta_{2}$ distributions of the field and overdensities are not likely to be drawn from the same population. However, we need to correct for the fact that we are searching for multiple correlations within the data, which increases the chances of a false positive result.

We use the Holm-Bonferroni (H-B) method (Holm 1979; see Appendix B of Kruijssen et al. 2019 for a recent astrophysical application) to check whether the difference in $\zeta$ distributions are robust against random fluctuations when searching for multiple different correlations within a dataset. We split the $p$-values shown in Figures 2 and 3 by test statistic, order the four $p$-values in increasing value, and label them $i=1, . ., 4$. We then test each $p$-value in increasing $i$ order against the $\mathrm{H}-\mathrm{B}$ criterion

$$
p_{i} \leq \frac{p_{\text {ref }}}{N_{\text {samp }}+1-i},
$$

where $N_{\text {samp }}=4$ is the number of samples and $p_{\text {ref }}=0.05$. In cases where the above condition is satisfied, the null hypothesis (that both distributions are drawn from the same underlying sample) can be rejected.

For the KS tests, the first two $p$-values $\left(p_{1}=1.4 \times 10^{-6} \leq\right.$ $\left.0.0167, p_{2}=8.9 \times 10^{-3} \leq 0.0125\right)$ pass the H-B criterion, so both the previously reported orbital period distributions and the $\zeta_{2}$ distributions are statistically different. Repeating this for the $\mathrm{AD}$ tests $\left(p_{1}=0.001 \leq 0.0125, p_{2}=0.04 \not \leq\right.$ 0.0167 ), we find that the orbital period distributions pass the H-B criterion but the $\zeta_{2}$ distributions do not. 

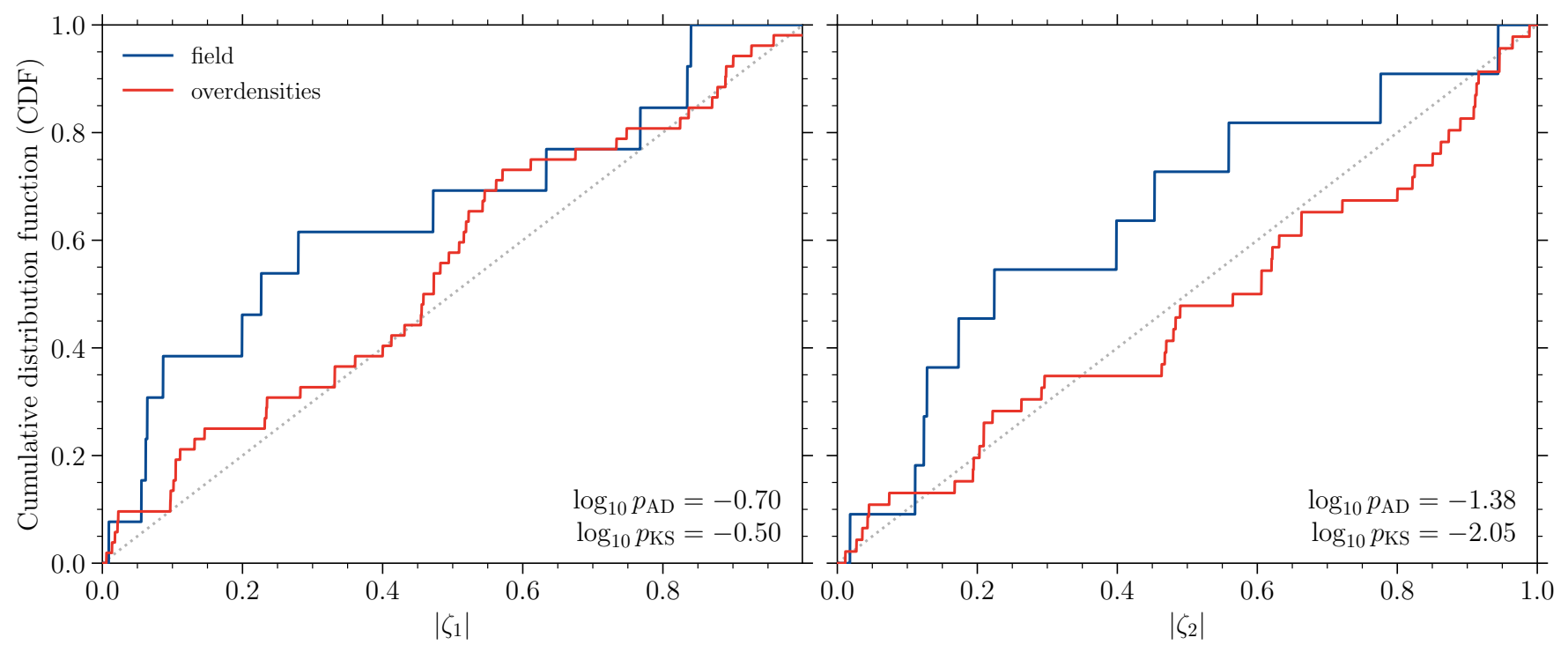

Figure 3. Cumulative distribution functions comparing the orbital period ratios of adjacent planet pairs to mean motion resonances (MMRs) for the field (blue) and overdensity (red) sub-samples from the full Winter et al. (2020) catalogue. The variables $\zeta_{1}$ (left panel) and $\zeta_{2}$ (right panel) quantify the difference between an observed period ratio and first-order and second-order MMRs, respectively, with $\zeta=0$ indicating that a pair coincides with an MMR. The dotted grey line shows the 1:1 relation between the CDF and $\zeta$ values for reference. The bottom-right corner of each panel shows the $p_{\mathrm{KS}}$ and $p_{\mathrm{AD}}$ values for two-sample Kolmogorov-Smirnov and Anderson-Darling tests, respectively, against the null hypothesis that the field and overdensity samples are drawn from the same $\zeta$ distributions. There is no statistically significant difference between the $\zeta_{1}$ distributions of the field and overdensity samples. However, the $\zeta_{2}$ distributions of the field and overdensities are not drawn from the same population. The orbital period ratios of adjacent planets in the field sample statistically lie closer to second-order MMRs than in the overdensity sample.

Given the $\zeta_{2}$ distributions are statistically different with one test statistic (KS) but not the other (AD), we opt to report the result as 'marginal'. Future work with improved data is required to determine whether the orbital period ratios of adjacent planets in the field sample statistically lie closer to second-order MMRs than in the overdensity sample. We note that repeating the analysis on the RV-only and transit-only samples shows that the detection method bias works in the opposite direction to the observed trend. In other words, correcting for this bias would likely strengthen the result that the $\zeta_{2}$ distributions are not drawn from the same underlying sample population.

In summary, the proximity of adjacent planet pairs to firstorder MMRs does not differ significantly between field and overdensity systems. A larger sample is needed to determine whether adjacent planet pairs in overdensities are found near second-order MMRs significantly less often than those in the field.

\subsection{Multiplicity: individual exoplanet surveys}

Finally, we return to investigating the difference in exoplanetary multiplicity between the systems in the field and overdensities, but now using sub-sets of the Winter et al. (2020) data. The goal of this exercise is to address the heterogeneity of the sample.

Rows 4 and 5 in Table 1 show the exoplanet multiplicity distributions of the field and overdensities when remov- ing systems with hot Jupiters (HJs; here defined by masses $>50 \mathrm{M}_{\oplus}$ and semi-major axes $<0.2 \mathrm{au}$ ) from the Winter et al. (2020) sample. The single-to-multiple ratio of the field and overdensities become statistically indistinguishable. This shows that the larger single-to-multiple planet ratio in overdensities compared to the field in the full sample is a direct consequence of HJs being (i) almost exclusively found in overdensities (Winter et al. 2020), and (ii) unlikely to have close companions (Steffen et al. 2012).

To see if this can fully explain the difference in multipicity between the field and overdensities, we then concentrate on data from the Winter et al. (2020) sample that are drawn from a single observational survey. This approach has several advantages. Firstly, the detection method for all sources is identical, so detection biases (such as RV versus transit) are not an issue. Secondly, the sample selection for the parent populations is identical. However, these advantages are offset by the fact that the number of planets in the field and overdensity samples are reduced.

Table 1 shows that no matter which individual survey is chosen, the single-to-multiple ratio is higher in overdensities than the field. However, the low number (or lack) of low density systems means that it is not possible to tell if this difference is statistically significant.

To investigate whether the lack of statistical significance might be caused by poorly constrained stellar parameters, we repeated the analysis using only the Kepler planet data, 


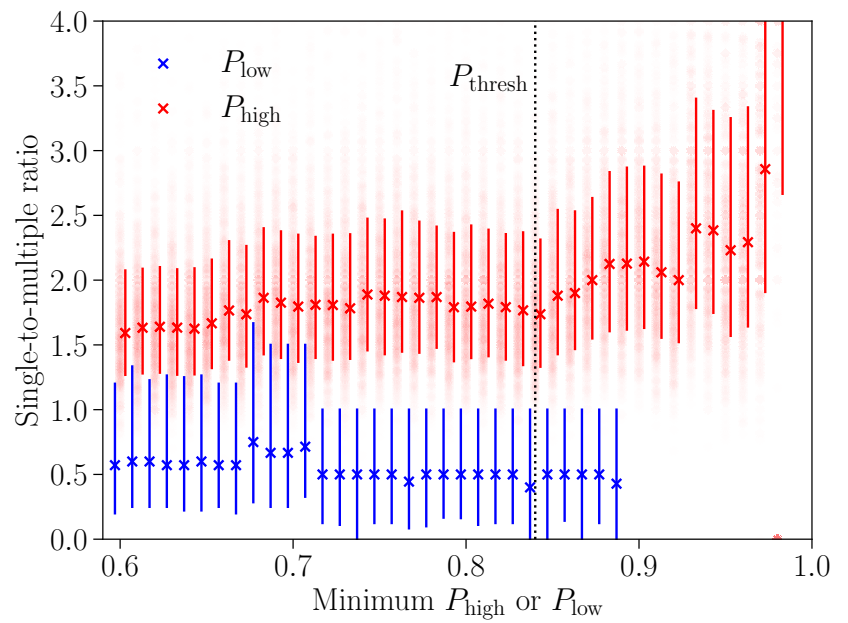

Figure 4. Ratio of single to multiple planet systems detected with Kepler as a function of the probability that the host star is in a high density $\left(P_{\text {high }}\right.$, red) or low density $\left(P_{\text {low }}\right.$, blue) environment. The crosses show the median single-to-multiple ratio at each $P_{\text {high }}$ and $P_{\text {low }}$ from 1000 Monte Carlo samples of the data drawn using Poisson statistics from the observed multiplicity. The error bars show the $16^{\text {th }}$ to $84^{\text {th }}$ percentile range of the Monte Carlo samples. The red, opacity-weighted circles show single Monte Carlo realisations for the high density sample. The vertical dotted line shows the fiducial threshold value of $P=0.84$ used to assign systems to overdensities or the field.

and replacing the stellar parameters in the NASA Exoplanet Archive with those from the California-Kepler Survey (Fulton \& Petigura 2018, denoted by 'CKS' in Table 1 and 2). We find that the single-to-multiple ratio contrast increases substantially, with overdensities having a factor 2.6 larger singleto-multiple ratio than the field. This increases to a factor of 3.5 when the lower mass cutoff of the stars is reduced from $0.7 \mathrm{M}_{\odot}$ (as in Winter et al. 2020 ) to $0.5 \mathrm{M}_{\odot}$, which ensures that all Kepler stars are included.

Figure 4 shows the results of a Monte Carlo experiment to determine the statistical robustness of this difference in multiplicity between the high and low-density Kepler samples. We generate 1000 synthetic planet populations by randomly drawing from Poisson distributions with mean numbers of planets given by the observed numbers of single and multiple planet systems in the low and high-density sub-samples, and repeat the multiplicity analysis. The error bars in Figure 4 show the range from the 16th to 84th percentile of the Monte Carlo realisations. We repeat the multiplicity analysis using different probability thresholds that a given system is in a high-density $\left(P_{\text {high }}\right)$ or low-density $\left(P_{\text {low }}\right)$ environment.

Figure 4 shows that for planetary systems taken from a single observational survey (Kepler) with a uniform selection criterion (i) there is a statistically significant difference between the single-to-multiple ratio between the high and low density samples, and (ii) that this difference increases as the probability that a given system is in a low or high-density environment increases. As the Kepler sample contains few HJs (Wright et al. 2012), we conclude that the difference in multiplicity between low and high-density environments extends to the full planet population, and is not restricted to HJs alone. We also repeat the analysis using different cuts in stellar parameters to verify that the distributions of host star properties between the field and overdensities are statistically indistinguishable (see, e.g. the bottom rows of Table 2).

In summary, no matter how the data are split, the same trend in planetary multiplicity between the low and highdensity sub-samples is recovered, although the degree of statistical significance weakens with decreasing sample size.

\section{DISCUSSION}

We now discuss what insight these observed differences in the field and overdensity planet populations may provide into the role that stellar clustering plays in the formation and evolution of planetary architectures. The planet multiplicity distribution around field stars provides a considerably better match to the multiplicity distribution of co-planar systems in the Mulders et al. (2018) models. Under the reasonable assumption that co-planar systems are the least likely to have been perturbed, the field systems represent an ideal subsample of exoplanetary systems to compare with simulations and models attempting to understand how planets form and evolve in effective isolation.

In contrast, we find a factor 1.6-2.0 excess of singleplanet systems in overdensities compared to the field for the full Winter et al. (2020) sample, and that this increases up to a factor 3.5 when only using planets detected by Kepler. This suggests that environments of high stellar phase space density play a prominent role in setting the planetary multiplicity, and may be a key contributing factor in the observed Kepler dichotomy. Stellar clustering may therefore play an important role in creating the two different populations of planetary architectures that previous studies (e.g. Lissauer et al. 2011; Hansen \& Murray 2013) have concluded are required to reproduce the observed exoplanet multiplicity distribution. Further comparison between the overdensity and field planet populations offers a fruitful new avenue to distinguish the relative influence of the different mechanisms postulated to be responsible for producing the excess of single-planet systems (e.g. Johansen et al. 2012; Hansen \& Murray 2013; Morton \& Winn 2014b; Ballard \& Johnson 2016).

The observed trend that the orbital periods of singleplanet systems in overdensities are smaller than the orbital periods of multiple-planet systems is consistent with previous studies showing that single-planet systems have shorter periods than systems hosting multiple planets (e.g. Weiss et al. 2018b, fig. 9). As this trend is far more pronounced in overdensities than in the field, whatever mechanism may be responsible for the period decrease in single-planet systems 
must be more effective when the host star resides in a higher density environment.

Having already concluded that entire planetary system architectures can be changed by the environment, the comparison between $\zeta_{1}$ and $\zeta_{2}$ for the field and overdensities probes the effect of the environment on mean motion resonances. As $\zeta_{1}$ between adjacent planet pairs is indistinguishable between the field and overdensities, first-order mean motion resonances must reform easily after disruption by environmental perturbations. Although the result is currently statistically marginal, the fact that the orbital period ratios of adjacent planets in the field may lie closer to second-order mean motion resonances (i.e. have lower $\zeta_{2}$ than overdensities) suggests that second-order resonances may reform less easily after environmental perturbations. Following this logic, second-order resonances could therefore either be imprints of the planet formation process, i.e. once they are destroyed they are not re-established, or they are never truly stable to perturbations and represent a transient state. In the latter case, the ratio between their formation and disruption timescales is higher in environmentally-perturbed systems than in environmentally unperturbed systems, causing their incidence to decrease.

Finally, we note a recent study by Adibekyan et al. (2021), who compare the orbital period distributions between overdensities and the field identified by Winter et al. (2020) for a small, but homogeneous sample of RV-detected planets with improved host stellar parameters, finding no significant difference. This result is consistent with our findings (as well as Extended Data Figure 8 of Winter et al. 2020), which show that (1) RV detections are generally too small to identify statistically significant differences and (2) the difference between overdensities and the field is largest for transit detections. Our statistical tests using the California-Kepler Survey sample (Fulton \& Petigura 2018) corroborate this interpretation, demonstrating that the environmental dependence of multiplicity is not dominated by the Jupiter-mass planets detected in RV surveys.
In summary, the above analysis of planetary multiplicity and orbital period distributions as a function of host stellar phase space density shows that ambient stellar clustering in the large-scale environment plays an important role in shaping the architecture of planetary systems. Understanding the demographics of the planet population at large will require linking the physical mechanisms acting on this wide variety of different scales, from planet formation and evolution to stellar dynamics and galaxy evolution.

\section{ACKNOWLEDGMENTS}

We thank the anonymous referee for insightful comments and suggestions which substantially improved the paper. We thank Paola Caselli, Trevor David, Laura Kreidberg, and Diego Turrini for helpful suggestions. M.C. and J.M.D.K. gratefully acknowledge funding from the Deutsche Forschungsgemeinschaft (DFG, German Research Foundation) through an Emmy Noether Research Group (grant number KR4801/1-1) and the DFG Sachbeihilfe (grant number KR4801/2-1). J.M.D.K. gratefully acknowledges funding from the European Research Council (ERC) under the European Union's Horizon 2020 research and innovation programme via the ERC Starting Grant MUSTANG (grant agreement number 714907). This research made use of data from the European Space Agency mission Gaia (http://www.cosmos.esa.int/gaia), processed by the Gaia Data Processing and Analysis Consortium (DPAC, http://www.cosmos.esa.int/web/gaia/dpac/consortium).

Funding for the DPAC has been provided by national institutions, in particular the institutions participating in the Gaia Multilateral Agreement. This research has made use of the NASA Exoplanet Archive, which is operated by the California Institute of Technology, under contract with the National Aeronautics and Space Administration under the Exoplanet Exploration Program.

Software: matplotlib (Hunter 2007), numpy (van der Walt et al. 2011), pandas (Reback et al. 2020), scipy (Jones et al. 2001), seaborn (Waskom et al. 2020)

\section{REFERENCES}

Adibekyan, V., Santos, N. C., Demangeon, O. D. S., et al. 2021, A\&A submitted, arXiv:2102.12346.

https://arxiv.org/abs/2102.12346

Ballard, S., \& Johnson, J. A. 2016, ApJ, 816, 66, doi: 10.3847/0004-637X/816/2/66

Borucki, W. J., Koch, D., Basri, G., et al. 2010, Science, 327, 977, doi: 10.1126/science.1185402

Borucki, W. J., Koch, D. G., Basri, G., et al. 2011, ApJ, 736, 19, doi: 10.1088/0004-637X/736/1/19
Cai, M. X., Portegies Zwart, S., \& van Elteren, A. 2018, MNRAS, 474, 5114, doi: 10.1093/mnras/stx3064

Chevance, M., Kruijssen, J. M. D., \& Longmore, S. N. 2021, ApJ submitted

Fang, J., \& Margot, J.-L. 2012, ApJ, 761, 92, doi: 10.1088/0004-637X/761/2/92

Fulton, B. J., \& Petigura, E. A. 2018, AJ, 156, 264, doi: 10.3847/1538-3881/aae828

Fulton, B. J., Petigura, E. A., Howard, A. W., et al. 2017, AJ, 154, 109, doi: 10.3847/1538-3881/aa80eb 
Gaia Collaboration, Prusti, T., de Bruijne, J. H. J., et al. 2016, A\&A, 595, A1, doi: 10.1051/0004-6361/201629272

Gaia Collaboration, Brown, A. G. A., Vallenari, A., et al. 2018, A\&A, 616, A1, doi: 10.1051/0004-6361/201833051

Hansen, B. M. S., \& Murray, N. 2013, ApJ, 775, 53, doi: 10.1088/0004-637X/775/1/53

He, M. Y., Ford, E. B., \& Ragozzine, D. 2019, MNRAS, 490, 4575, doi: 10.1093/mnras/stz2869

He, M. Y., Ford, E. B., Ragozzine, D., \& Carrera, D. 2020, arXiv e-prints, arXiv:2007.14473. https://arxiv.org/abs/2007.14473

Holm, S. 1979, Scandinavian Journal of Statistics, 65, 6

Hunter, J. D. 2007, Computing In Science \& Engineering, 9, 90, doi: 10.1109/MCSE.2007.55

Johansen, A., Davies, M. B., Church, R. P., \& Holmelin, V. 2012, ApJ, 758, 39, doi: 10.1088/0004-637X/758/1/39

Jones, E., Oliphant, T., Peterson, P., et al. 2001, SciPy: Open source scientific tools for Python. http://www.scipy.org/

Kennedy, G. M., \& Wyatt, M. C. 2013, MNRAS, 433, 2334, doi: 10.1093/mnras/stt900

Kruijssen, J. M. D., Longmore, S. N., \& Chevance, M. 2020, ApJ, 905, L18, doi: 10.3847/2041-8213/abccc3

Kruijssen, J. M. D., Pfeffer, J. L., Crain, R. A., \& Bastian, N. 2019, MNRAS, 486, 3134, doi: 10.1093/mnras/stz968

Lissauer, J. J., Ragozzine, D., Fabrycky, D. C., et al. 2011, ApJS, 197, 8, doi: 10.1088/0067-0049/197/1/8

Morton, T. D., \& Winn, J. N. 2014a, ApJ, 796, 47, doi: 10.1088/0004-637X/796/1/47

—. 2014b, ApJ, 796, 47, doi: 10.1088/0004-637X/796/1/47

Mulders, G. D., Pascucci, I., Apai, D., \& Ciesla, F. J. 2018, AJ, 156, 24, doi: 10.3847/1538-3881/aac5ea

NASA Exoplanet Archive. 2020, Composite Planet Data Table, IPAC, doi: 10.26133/NEA2

Reback, J., McKinney, W., jbrockmendel, et al. 2020, pandas-dev/pandas: Pandas 1.0.3, v1.0.3, Zenodo, doi: 10.5281/zenodo.3715232
Rogers, J. G., \& Owen, J. E. 2020, MNRAS submitted, arXiv:2007.11006, arXiv:2007.11006.

https://arxiv.org/abs/2007.11006

Sandford, E., Kipping, D., \& Collins, M. 2019, MNRAS, 489, 3162, doi: 10.1093/mnras/stz2350

Steffen, J. H., Ragozzine, D., Fabrycky, D. C., et al. 2012, Proceedings of the National Academy of Science, 109, 7982, doi: 10.1073/pnas.1120970109

van der Walt, S., Colbert, S. C., \& Varoquaux, G. 2011, Computing in Science and Engg., 13, 22, doi: 10.1109/MCSE.2011.37

Van Eylen, V., Albrecht, S., Huang, X., et al. 2019, AJ, 157, 61, doi: 10.3847/1538-3881/aaf22f

Waskom, M., Botvinnik, O., Ostblom, J., et al. 2020, mwaskom/seaborn: v0.10.0 (January 2020), Zenodo, doi: 10.5281/zenodo.3629446

Weiss, L. M., Marcy, G. W., Petigura, E. A., et al. 2018a, AJ, 155, 48, doi: 10.3847/1538-3881/aa9ff6

Weiss, L. M., Isaacson, H. T., Marcy, G. W., et al. 2018b, AJ, 156, 254, doi: 10.3847/1538-3881/aae70a

Winn, J. N., \& Fabrycky, D. C. 2015, ARA\&A, 53, 409, doi: 10.1146/annurev-astro-082214-122246

Winter, A. J., Kruijssen, J. M. D., Longmore, S. N., \& Chevance, M. 2020, Nature, 586, 528

Wright, J. T., Marcy, G. W., Howard, A. W., et al. 2012, ApJ, 753, 160, doi: 10.1088/0004-637X/753/2/160

Wright, J. T., Upadhyay, S., Marcy, G. W., et al. 2009, ApJ, 693, 1084, doi: 10.1088/0004-637X/693/2/1084

Zhu, W. 2020, AJ, 159, 188, doi: 10.3847/1538-3881/ab7814

Zinzi, A., \& Turrini, D. 2017, A\&A, 605, L4, doi: 10.1051/0004-6361/201731595 